INPLASY

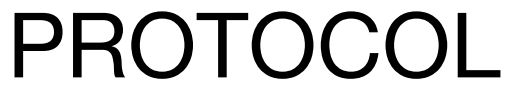

To cite: Hu et al. Survival and Prognostic Factors in Patients with Connective Tissue

Diseases Associated

Pulmonary Arterial

Hypertension: A Systematic

Review and Meta-analysis.

Inplasy protocol 202080005.

doi:

10.37766/inplasy2020.8.0005

Received: 03 August 2020

Published: 03 August 2020

Corresponding author:

Ai Cui

bj5453@sina.com

Author Affiliation:

Department of Pulmonary

and Critical Care Medicine,

Beijing Chao-Yang Hospital,

Capital Medical University

Support: Beijing Municipal

Hospital

Review Stage at time of this submission: Piloting of the study selection process.

Conflicts of interest:

All authors declare to have no real or perceived conflicts of interest with respect to this manuscript.

\section{Survival and Prognostic Factors in Patients with Connective Tissue Diseases Associated Pulmonary Arterial Hypertension: A Systematic Review and Meta-analysis}

Hu, Y1; Zhang, F2; Zhang, H³; Sheng, X; Huang, L5; Cui, A6.

Review question / Objective: This study aims to compare the survival rates between SSc-PAH and non-SSc-CTD-PAH as well as to determine the prognostic factors in CTD-PAH patients.

Condition being studied: Pulmonary arterial hypertension (PAH) is a rare complication and severe manifestation of connective tissue diseases (CTDs). The survival rate of nonSSc CTD-associated PAH (non-SSC-CTD-PAH) is better than that of SSc-PAH patients. Previous studies have reported prognostic factors for mortality in CTD-PAH. So far, only a few studies have paid attention to the integrated survival rates and the prognostic factors in CTD-PAH patients using metaanalysis. To fill in the gaps in the literature, our study aims to compare the 1-, 2-, 3-, and 5- survival rates between SSc-PAH and non-SSC-CTD-PAH as well as to determine the potential prognostic factors in CTD-PAH patients.

INPLASY registration number: This protocol was registered with the International Platform of Registered Systematic Review and Meta-Analysis Protocols (INPLASY) on 03 August 2020 and was last updated on 03 August 2020 (registration number INPLASY202080005).

\section{INTRODUCTION}

Review question / Objective: This study aims to compare the survival rates between SSc-PAH and non-SSc-CTD-PAH as well as to determine the prognostic factors in CTD-PAH patients. 
Condition being studied: Pulmonary arterial hypertension (PAH) is a rare complication and severe manifestation of connective tissue diseases (CTDs). The survival rate of non-SSc CTD-associated PAH (non-SScCTD-PAH) is better than that of SSc-PAH patients. Previous studies have reported prognostic factors for mortality in CTDPAH. So far, only a few studies have paid attention to the integrated survival rates and the prognostic factors in CTD-PAH patients using meta-analysis. To fill in the gaps in the literature, our study aims to compare the 1-, 2-, 3-, and 5- survival rates between SSC-PAH and non-SSC-CTD-PAH as well as to determine the potential prognostic factors in CTD-PAH patients.

\section{METHODS}

Search strategy: Two of the authors searched Pubmed, EMBASE and Cochrane Central Register databases for relevant articles. The key search words were ("Connective Tissue Diseases" OR "Systemic Lupus Erythematosus" OR "Mixed Connective Tissue Disease" OR "Systemic Scleroderma") AND ("Pulmonary Hypertension" OR "Pulmonary Arterial Hypertension").

Participant or population: CTD-PAH patients. SSc, SLE, pSS, and MCTD were diagnosed by American College of Rheumatology diagnostic criteria, 2002 international classification criteria, and 1987 Sharp criteria. PAH was confirmed by right heart catheterization (RHC) and could be classified into WHO group 1.

Intervention: This meta-analysis will include observational studies without having the intervention methods.

Comparator: This meta-analysis will include observational studies without having the comparator treatments.

Study designs to be included: Cohort studies or case-control studies.

Eligibility criteria: Studies contained patients suffering from SSc, SLE, pSS, and MCTD associated PAH. Studies were excluded due to: (1) Patients enrolled with interstitial lung disease (ILD). (2) PAH diagnosed only by echocardiography. (3) Studies aimed to determine the diagnostic value or treatment effects. (4) CTD-PAH correlated with juvenile, pregnancy, and lung transplantation. If patients in cohorts were republished, we would choose the studies that reported the most comprehensive data with longer study periods.

Information sources: Pubmed, EMBASE and Cochrane Central Register databases.

Main outcome(s): The outcomes comprised survival rates or prognostic factors such as cardiac function (NYHA or WHO Functional class and 6MWD), hemodynamics analysis (mRAP, mPAP, PCWP, CO, Cl, PVR), pulmonary function tests (FVC $\%$ predicted, TLC \% predicted, FEV1 \% predicted, DLco $\%$ predicted), echocardiography (pericardial effusion).

Quality assessment / Risk of bias analysis: Newcastle Ottawa scale (NOS) was used to assess the risk of bias in the included studies. NOS consists of three subscales: patient selection, comparability, and assessment of outcome. A study can have a score of 0 to 9 in NOS, where the higher the score is, the better the study's quality is. A study of high quality which scored at 6 or above would be finally included.

Strategy of data synthesis: Statistical analysis for survival rates and HRs of prognostic factors were performed using STATA.

Subgroup analysis: For calculating survival rates, we will divide studies into subgroups (SSc-PAH and non-SSc-CTD-PAH).

Sensibility analysis: We will use STATA 15 (db metaninf) to perform sensibility analysis.

Language: The language was restricted to English.

Country(ies) involved: China. 
Keywords: Connective tissue diseases; pulmonary arterial hypertension; survival; prognostic factors; meta-analysis.

Contributions of each author:

Author 1 - Yuqi Hu.

Author 2 - Hui Zhang.

Author 3 - Fan Zhang.

Author 4 - Xiaowen Sheng.

Author 5 - Liru Huang.

Author 6 - Ai Cui. 